\title{
Development of Dark-Field Photonic Scanning Transmission Electron Microscopy (DFP-STEM)
}

Atsushi Muto ${ }^{1}$, Yukari Dan ${ }^{2}$ and Kotaro Hosoya ${ }^{3}$

${ }^{1}$ Hitachi High-Tech America, Inc., Clarksburg, Maryland, United States, ${ }^{2}$ Hitachi High-Tech Europe GmbH, Warrington, England, United Kingdom, ${ }^{3}$ Hitachi High-Tech Canada, Inc., Toronto, Ontario, Canada

Scanning transmission electron microscopy (STEM) utilizing a scanning electron microscope (SEM) is widely used in the fields of semiconductor production and nanomaterial analysis as well as biological / soft material applications for surface imaging coupled with compositional information of internal fine structures.

In addition to bright-field STEM (BF-STEM), dark-field STEM (DF-STEM) is rapidly becoming a more popular imaging approach in SEM due to the increasing needs for correlative imaging of internal composition as well as surface/structural information in order to obtain accurate and quick characterization of materials. To address these requirements, remarkable developments of DF-STEM detection systems, methodology and applications have been developed in the last several years [1].

Traditionally, there have been two major approaches for DF-STEM imaging by SEM. The first technique is to utilize a dedicated DF-STEM electron detector and signal processing system, which has been adopted in ultra-high resolution SEMs for imaging and analysis of angstrom-level structures [2]. The second technique utilizes the 'conversion method' [3]. In this approach, the scattered electron signal is converted to a secondary electron (SE) and then detected by a conventional SE detector. This allows for STEM detection capability that is easily obtained with no hardware modification, however, technical difficulties including low yield originating from loss of signal during reflective conversion and substantial noise from normal SE signals present issues. To resolve these technical difficulties, we reported a novel conversion type BF-STEM holder using a combination of photonic detection via a scintillator-based reflector in conjunction with a CL detector instead of conversion plate [4].

In this study, a novel STEM imaging concept deemed Dark-Field Photonic Scanning Transmission Electron Microscopy (DFP-STEM) was implemented using a redesigned scintillator-based reflector with CL detector for DF-STEM observation utilizing photons instead of electrons. Figure 1 demonstrates a schematic diagram of this novel STEM observation approach. By optimizing the sample holder design and scintillator conversion plate architecture to detect scattered electrons, DF-STEM signals were able to be visualized with a photon detector. Today, CL-type technology, such as the ultra-variable detector (UVD), are commonly used in modern variable pressure SEMs [5]. The Hitachi UVD functions as a sole photon detector, discriminating SE detection by changing imaging parameters without any hardware modification.

Figure 2 shows simultaneous imaging of carbon nanotubes (CNTs) with SE, dedicated BF-STEM, and DFP-STEM. The Hitachi SU7000 schottky SEM was used to obtain images with an accelerating voltage of $30 \mathrm{kV}$. In the SE image, fine topographic surface information is easily observed (a). Internal sample information such as the inner tube diameter and compositional information are, however, mixed in BFSTEM image (b). Distributions of metal catalysts in/around CNTs were highlighted in new DFP-STEM imaging method with signal intensity being sufficient to confirm fine particles smaller than $10 \mathrm{~nm}$ in diameter. Additionally, a comparison of signal yield for the conventional conversion method and 
simultaneous STEM imaging applications of various types of materials will also be reported to verify the benefit of this new DFP-STEM methodology.

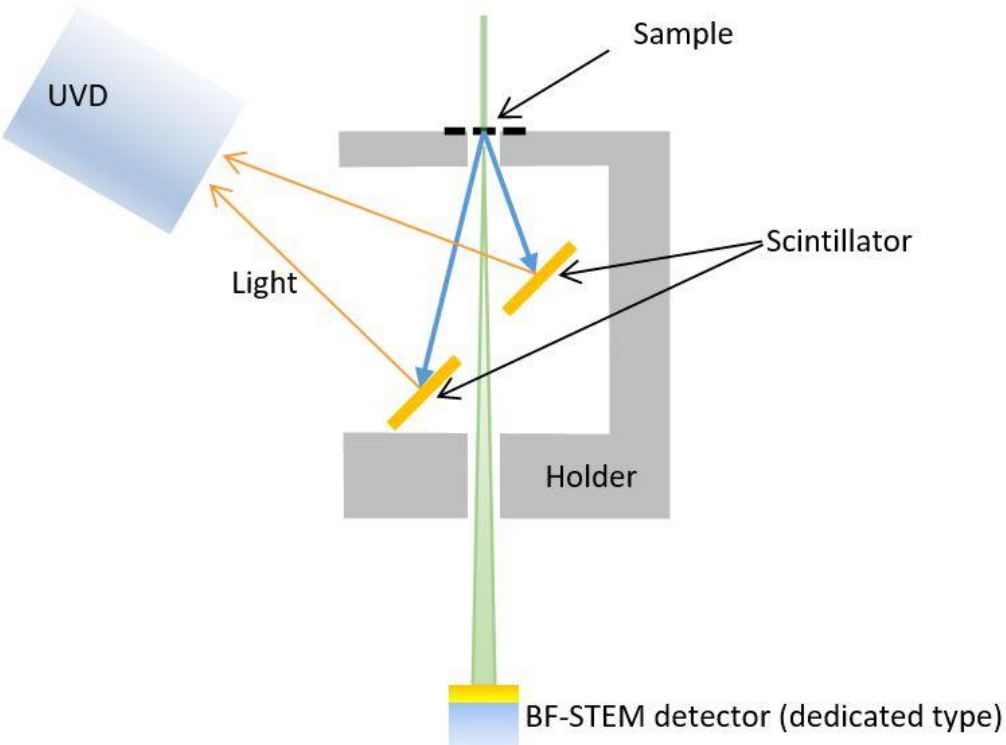

Figure 1. Schematics of photon detection based DFP-STEM holder with simultaneous BF-STEM
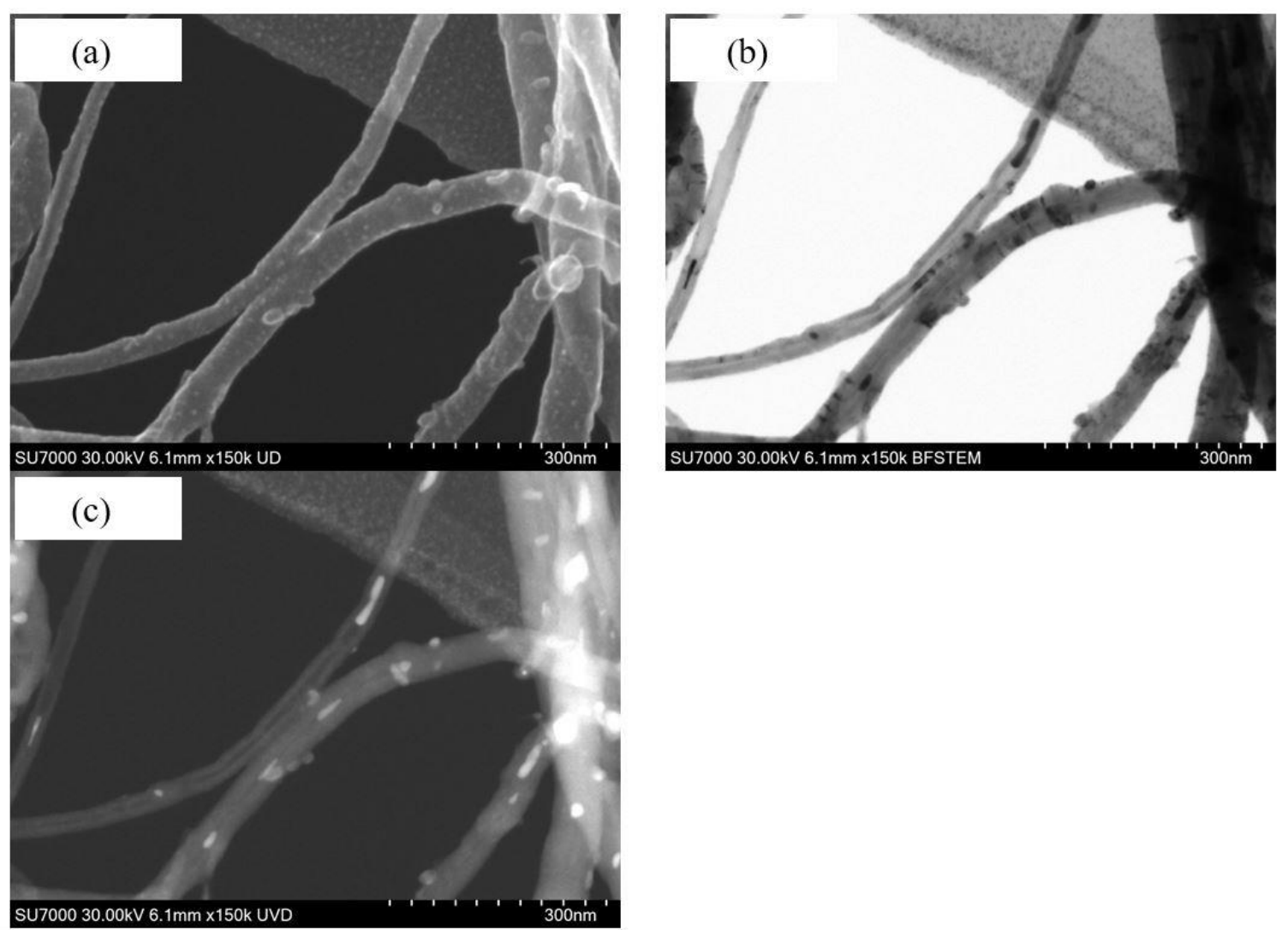

Figure 2. Imaging of CNTs with simultaneous signal detection of SE, BF-STEM (Dedicated) and DFPSTEM (new scintillator-based method). (a) SE image (b) BF-STEM image using dedicated STEM (c) DFP-STEM image using new STEM scintillator signal conversion 


\section{References}

[1] N Sakamoto et al, Microsc.Miacroanal.19 (Suppl 2) (2013) p.p.386

[2] Y Orai et al, J. Phys.: Conf. Ser (2014) p.p.522

[3] Joseph Goldstein et al. in "Scanning Electron Microscopy and X-ray Microanalysis", (2003) p.p.203.

[4] K Hosoya et al, Microsc.Miacroanal.25 (Suppl 2) (2019) p.p.552

[5] M Nishimura et al, Hitachi S.I.NEWS Vol.56 No.1 (2013) p.p.37-41 